\begin{tabular}{l} 
Sharif University of Technology \\
Scientia Iranica \\
SCIENTIA \\
IRAN \\
Thansactions A: Civil Engineering \\
\hline
\end{tabular}

\title{
Employing nonlinear dynamic concepts for catchment classification using runoff response of catchments
}

\author{
H. Delafrouz ${ }^{a, *}$ A. Ghaheri ${ }^{a}$, and M.A. Ghorbani ${ }^{b}$ \\ a. School of Civil Engineering, Iran University of Science and Technology, Tehran, Iran. \\ b. Department of Water Engineering, University of Tabriz, Tabriz, Iran. \\ Received 25 September 2016; received in revised form 12 November 2016; accepted 31 December 2016
}

\author{
KEYWORDS \\ Catchment \\ classification; \\ Chaos theory; \\ Correlation \\ dimension; \\ System complexity; \\ Streamflow variability.
}

\begin{abstract}
Classification is considered as a fundamental step towards improved science and management data. Introducing methods that describe the underlying dynamics of runoff could be a promising way for catchment classification. In this respect, chaos theory and correlation dimension were applied to test its ability to construct a concept to introduce a catchment classification framework in this study. The correlation dimension, as an indicator, was calculated for the daily river flow of sixty grouping stations in different catchments in Iran, ranging in size from 8 to $36500 \mathrm{~km}^{2}$. The results confirmed that applying this indicator to catchments in varied ranges, from low to high complexity, could also be classified. The results showed that Iran's catchments could be classified into four groups based on the complexity degree of runoff time series. The group is categorized as follows: low dimension $\left(D_{2}<=4\right)$ as Group 1 , medium dimension $\left(D_{2}=5\right)$ as Group 2 , high dimension $\left(D_{2}=>6\right.$ ) as Group 3, and unidentifiable as Group 4. The spatial pattern classification of Iran's catchments indicates that catchments with different climate characteristics, which are located at a far distance from each other, might yield similar responses along with the same level of complexity.
\end{abstract}

(C) 2018 Sharif University of Technology. All rights reserved.

\section{Introduction}

Integrated catchment management is of great importance to manage the resulted runoff of rainfalls. Several attempts have been made to develop new approaches to hydrologic planning and watershed management purposes. Moreover, the invention of powerful computers and measurement devices leads to a more rapid and significant growth of these approaches. Despite all these attempts, the hydrological modelling state has not changed significantly and seems to need more investigations. Although the model complexity pre-

\footnotetext{
*. Corresponding author. Tel.: +9821 66906467; Fax: +982166906468

E-mail address: delafrooz@gmail.com (H. Delafrouz)
}

sented is increasing, there are no significant changes in its efficiency and accuracy. As stated by McDonnell and Woods [1], there is no clear guidance to verify which model or model structure is suitable for each catchment type or management questions. In other fields of science, this problem is solved by classification concepts such as Carolus Linnaeus' organism classification system in biology, the periodic table in chemistry, or dimensionless numbers in fluid dynamics. Gani et al. studied 45 methods for classifying "big data" [2]. Basically, the classification can provide valuable information and is one of the measures of the science maturity [3]. A classification framework in which each catchment increases the understanding of the definition of catchment functions is necessary [4]. Therefore, an appropriate classification framework can be beneficial in catchment management to develop and apply a suitable model in hydrology science. 
Two general approaches to catchment classification are categorized as deductive and inductive [5]. The deductive approach is based on studying and analysing the effects of the environmental influences (such as catchments' physical, hydrological, and climatic similarities) on discharge. In the inductive approach, hydrologic time series are analysed directly to identify the "complexity" (see [6] for further information) and dynamic pattern properties of discharge (see Figure 1).

According to the complexity of the rainfall-runoff phenomenon, catchments show a varied range of response behaviours, even if they are similar to or situated near each other. The effects of all factors on the catchment system are embedded in the discharge time series, reflecting both climatic and morphologic characteristics. Therefore, in cases where time series data are available, the inductive approach seems to be more suitable to find catchments' similarities and classification.

There are two main procedures in the inductive approach. The first procedure is based on statistical methods, using data properties such as size, frequency, duration of seasons, and variation of flow regime characteristics (i.e., seasonal pattern, frequency and duration of droughts, floods, annual runoff variability, and variation rate) $[5,7-10]$.

The second procedure is based on the application of nonlinear dynamics and complexity concepts to discharge time series. Zoppou et al. [11] applied wavelet spectral analysis to some Australian runoff stations and classified the basins into twelve categories using the spectral properties of the outflow time series. Krasovskaia [12] classified the hydrologic time series in Scandinavia using the entropy concept. Sen [13] used Lempel-Ziv Complexity (LZC) measures to analyse the complexity of monthly runoff data in England, and proposed that the catchments can be classified using this measure. Chou [14] applied sample entropy (SampEn) to rainfall and runoff time series to study the evolution of complexity of different temporal scales.

To introduce the concepts of chaos theory, as well as the reconstruction of the phase-space in hydrology, together with the similarities between the hypothetic and hydrologic phenomena such as rainfallrunoff, a number of researchers have proposed the use of this theory for classification. Sivakumar [15] proposed the use of nonlinear concepts to identify the number of dominant processes in hydrologic systems. Sivakumar et al. [16] showed the efficiency of phasespace reconstruction in identifying deterministic and random time series with two artificial time series and proposed the use of this approach to catchment classification. They also analysed and tested this idea for daily and monthly streamflow time series under varying conditions. Sivakumar and Singh [6] classified a number of catchments in the western United States by reconstructing the phase-space and employing the correlation dimension $\left(D_{2}\right)$ of monthly discharge time series. They classified the discharge of the catchments into four categories based on the following correlation dimension values: low dimension $\left(D_{2}<3\right)$, medium dimension $\left(3<D_{2} \leq 6\right)$, high dimension $\left(D_{2}>\right.$ $6)$, and unidentifiable ( $D_{2}$ not identifiable). They recommended testing this approach in other parts of the world with different time scales for future researches. Sivakumar et al. [17] classified a number of monthly rainfall time series in Western Australia into five groups based on correlation dimension values. Vingnesh et al. [18] employed false nearest neighbours to study spatial variability of streamflow across the United States.

During recent years, there has been an increasing interest in developing a framework for catchment classification; thus, a number of attempts have been made to reach a more efficient modelling (see [4,6,18-20]). Our investigation of the literature indicates that only a few studies have investigated the spatial variation of discharge data at different locations with spatial climatic variability through employing the concept of chaos theory (see [6] and [21] as examples). To the best of authors' knowledge, there is only one study in which Vignesh et al. [18] used the chaos method to classify the United States catchments over the whole area of the USA. Therefore, we believe that the present study is suitable due to the number and distribution of data tested. In addition, since Iran is a developing country where there are obviously problems with recorded data, this study can appropriately identify this classification pattern.

The results of this research can be used to

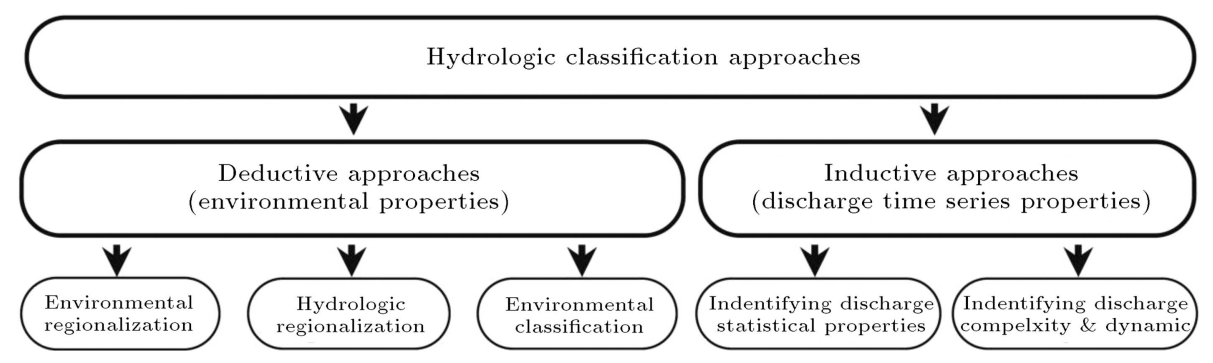

Figure 1. General categories of catchment classification study. 
translate large volumes of information into smaller and homogeneous volumes for easy modelling, complete missing/incomplete data, and expanding point information to regional information for ungauged sites, using raw data instead of index or other criteria, grading basins using the basins' functions instead of flow regimes, easily determining the complexity of stream flows using the nonlinear dynamic method in order to classify the basins and suggest appropriate models, and determining suitable basins for the appropriate models and for homogeneous basins.

This approach is used to classify the catchments in the whole area of Iran with different climates across the entire country. The mentioned method is applied to daily discharges of sixty stations, which are spatially distributed all over the country. Since Iran is subject to various climates, the country's catchments have been selected to examine the ability of the chaos concept for different climatic catchment classifications.

This study aims to present a classification pattern based on chaos theory and dynamic phase-space reconstruction. For this purpose, the following steps are proposed:

a) Employ the phase-space reconstruction concept and calculate the correlation dimension $\left(D_{2}\right)$;

b) Evaluate $D_{2}$ as a criterion for measuring the complexity and classification;

c) Present a pattern for the classification (identify the limit of group).

In the next sections of the paper, the methodology including dynamic phase space, average mutual information, and correlation dimension is described. Subsequent sections present the study area and data. The results obtained via the proposed modelling framework are then presented and discussed, followed by the conclusion.

\section{Reconstruction of dynamic phase space}

The concept of phase space is a useful tool for conducting research into the dynamic systems, and several researchers have used this concept to study various hydrological phenomena in the past two decades [22]. According to this concept, a dynamic system can be represented by a set of effective variables influencing the system's behaviour and is modelled by a phase space diagram, in which each point represents the system's behaviour at a specific time. Takens [23] proposed the common method. According to this method, given one single-variable time series present in a system, namely $\mathrm{Xt}$, the state vector can be reconstructed as follows:

$$
Y_{t}=\left(X_{t}, X_{t-\tau}, X_{t-2 \tau}, \ldots, X_{t-(m-1) \tau}\right),
$$

where $m$ is the dimension of vector $Y_{t}$, called embedding dimension, $\tau$ is referred to as the delay time and is usually used as an appropriate multiple of the sampling interval for discrete time series. Dimension $m$ can be considered as the minimum number of variables required to represent the system.

The Grassberger-Procaccia method [24], which is one of the common methods for embedding dimension estimation and chaos identification, is used in this study. Average Mutual Information (AMI) and Auto Correlation Function (ACF) methods are two typical methods used to estimate the delay time. Since the runoff generation of a catchment is a complex and nonlinear process, it is better to use a non-linear method to estimate the delay time. Therefore, the AMI method, which has a non-linear structure, is used to calculate the delay time in this study. A brief description of the AMI and correlation dimension methods is presented below.

\subsection{Average mutual information}

This method measures the amount of information about the value of $X(t+\tau)$ if the value of $X(t)$ is known [25]. For a discrete time series, with $X(t)$ and $X(t+\tau)$, the Average Mutual information (AMI), $I(\tau)$, is defined as follows:

$$
\begin{aligned}
I(\tau)= & \sum_{X(t), X(t-\tau)} P(X(t), X(t+\tau)) \\
& \times \ln \left[\frac{P(X(t), X(t+\tau))}{P(X(t)) \times P(X(t+\tau))}\right],
\end{aligned}
$$

where $P(X(t))$ and $P(X(t+\tau))$ are individual probabilities of $X(t)$ and $X(t+\tau)$, respectively, and $P(X(t), X(t+\tau))$ is the joint probability density. In this method, the time of the first minimum in average mutual information function is defined as the optimum delay time.

\subsection{Correlation dimension}

The correlation dimension method is also named as correlation integral analysis. Correlation integral $C(r)$ is calculated using Grassberger-Procaccia approach [24] as the common method. According to this method, for an $m$-dimensional phase space, correlation integral $C(r)$ is defined as follows:

$$
C(r)=\lim _{N \rightarrow \infty} \frac{2}{N(N-1)} \sum_{\substack{i, j \\(1 \leq i<j \leq N)}} H\left(r-\left\|Y_{i}-Y_{j}\right\|\right),
$$

where $H$ is the Heaviside function, with $H(u)=1$ for $u>0$ and $H(u)=0$ for $u \leq 0$ in which $u=r-$ $\left|Y_{i}-Y_{j}\right|, r$ is the radius of the sphere centred on $Y_{i}$ or $Y_{j}$, and $N$ is the number of points on the reconstructed attractor. If the time series is described as an attractor, 
for positive values of $r$, correlation function $C(r)$ and $r$ are related to each other as follows:

$$
C(r)=\alpha r^{D_{2}}
$$

where $\alpha$ is a constant value, and $D_{2}$ is a correlation exponent or the slope of $\ln C(r)$ versus $\ln r$ given by:

$$
D_{2}=\lim _{r \rightarrow 0} \frac{\ln C(r)}{\ln r} .
$$

This gradient is generally calculated by fitting a line into the main range of $r$ (i.e., scaling range). The type of the system can be determined by studying the behaviour of $D_{2}$ versus dimension $m$. If $D_{2}$ varies linearly with increasing $m$ without achieving a saturation value, the system can be considered as a stochastic system. In contrast, if $D_{2}$ is saturated at a definite value of $m$, the system is considered as a deterministic system [25].

\section{Data sets}

The study area is the whole area of Iran consisting of different climate catchments. Iran's territory is divided into six main catchments: Sarakhs, Caspian Sea, Orumieh Lake, Persian Gulf, Gulf of Oman, and Central Basin. The climate in each catchment is completely different from the other. The average rainfall in these catchments varies from $337 \mathrm{~mm} /$ year in the Caspian Sea catchment to $102 \mathrm{~mm} /$ year in the Oman Sea catchment. In this study, the catchment areas vary from $8 \mathrm{~km}^{2}$ (station 19-148 at Ardebil province) to $36500 \mathrm{~km}^{2}$ (station 24-029 at Bushehr province). The daily recorded runoff data ranging from 18 to 60 years recorded in 60 synoptic stations are used in this study (see Figure 2). As shown in Table 1, the properties of streamflow, such as maximum and average, are of high variation values.

The spatial distribution of the selected stations and their climate properties are shown in Figure 2. Table 1 shows the elevation, area, and outflow properties of each station. As seen from Figure 2 and Table 1, a wide range of topography, land use, geology, soil types, and climate data are used in this study. The minimum and maximum elevations are -23 and 2068 meters, respectively. The basin areas range from 8 to $36500 \mathrm{~km}^{2}$. The maximum flow rate is between 0.95 and $2866 \mathrm{~m}^{3} / \mathrm{s}$, and the average flow rate is between 0.07 and $48.33 \mathrm{~m}^{3} / \mathrm{s}$.

\section{Results and discussion}

The correlation dimension of each time series in each station is calculated using the Grassberger-Procaccia method (Table 1). The comments of some researchers in the calculations of the correlation dimensions are considered in this study [26-29]. Three stations (21$163,21-966$, and 19-055) are selected as examples to illustrate the correlation dimension.

Figure 3 shows how the scalar time series is reconstructed in a higher dimensional phase-space, according to Eq. (1), to represent the underlying dynamics. In fact, Figure 3 represents the phase-space diagram in two dimensions $(m=2)$ with a delay time of $\tau=1$, i.e. the projection attractor on the plane $\left(X_{i}, X_{i+1}\right)$. As seen in Figure 3 , while the projection produces a well-defined structure attractor in station 19-055 (Figure 3(c)), the structures of station 21-163 are scattered in the phase space (Figure 3(a)). The state of station 21-966 is approximately between the other two stations (Figure 3(b)). It should be noted that, according to the presented projections in Figure 3, the complexity of the system in station $19-055$ is less than that of station 21-163. Therefore, station 19055 can be an approximate deterministic system, while station $21-163$ is a stochastic one.

This result is verified using the correlation dimension results, discussed in the following step.

The delay time is estimated using the AMI method. Then, the correlation function of each time series is calculated using the embedding dimensions $(m)$ values from 1 to 40 and the calculated delay time. Figure 4 shows the relationship between correlation function $C(r)$ and radius $r$ for the increasing values of $m(\log C(r)$ versus $\log r)$. In Figure 4, there is a middle region where correlation function $C(r)$ starts to stray from linearity. The correlation function from these regions is calculated by least square error method, as shown in Figure 5. Moreover, the relationship between the correlation exponent and the embedding dimension values is shown in Figure 5.

As seen in Figure 5, while the correlation exponent values increase with embedding dimension values without any bound in station 21-163, the correlation exponent is saturated in a finite value in station 19055. The infinite increase of the correlation exponent in station 21-163 is an indicator of stochastic behaviour in the streamflow time series. In contrast, the correlation dimension in station 19-055 is saturated at $D_{2}=3$, suggesting the possible presence of the chaotic behaviour in the streamflow time series. In station 21-966, the correlation dimension is equal to 5 ; as can be observed in Figure 3, the behaviour of this station is relatively similar to those of the other two stations. The results of the other investigated stations are presented in Table 1. The sign " $\infty$ " in the table shows the indefinability of the correlation dimension parameter.

According to Table 1, the correlation dimension values have a wide range of variation between 3 (very small) and $\infty$. Considering stations 29-011 and 12033 with undefined values of $D_{2}$, these stations are located far from each other and in two different 
Table 1. Details and results of stations' analysis.

\begin{tabular}{|c|c|c|c|c|c|c|c|c|c|c|c|}
\hline \multirow[b]{2}{*}{ Station } & \multicolumn{2}{|c|}{$\begin{array}{l}\text { Catchment } \\
\text { properties }\end{array}$} & \multicolumn{2}{|c|}{$\begin{array}{c}\text { Outflow } \\
\text { properties }\end{array}$} & \multirow[b]{2}{*}{$D_{2}$} & \multirow[b]{2}{*}{ Station } & \multicolumn{2}{|c|}{$\begin{array}{l}\text { Catchment } \\
\text { properties }\end{array}$} & \multicolumn{2}{|c|}{$\begin{array}{c}\text { Outflow } \\
\text { properties }\end{array}$} & \multirow[b]{2}{*}{$D_{2}$} \\
\hline & $\begin{array}{c}\text { Elevation } \\
(\mathrm{m})\end{array}$ & $\begin{array}{l}\text { Area } \\
\left(\mathrm{km}^{2}\right)\end{array}$ & $\begin{array}{c}\text { Average } \\
\left(\mathrm{m}^{3} / \mathrm{s}\right)\end{array}$ & $\begin{array}{c}\operatorname{Max} \\
\left(\mathrm{m}^{3} / \mathrm{s}\right)\end{array}$ & & & $\begin{array}{c}\text { Elevation } \\
(\mathrm{m})\end{array}$ & $\begin{array}{c}\text { Area } \\
\left(\mathrm{km}^{2}\right)\end{array}$ & $\begin{array}{c}\text { Average } \\
\left(\mathrm{m}^{3} / \mathrm{s}\right)\end{array}$ & $\begin{array}{c}\operatorname{Max} \\
\left(\mathrm{m}^{3} / \mathrm{s}\right)\end{array}$ & \\
\hline $12-033$ & 100 & 114.5 & 0.39 & 67 & $\infty$ & 21-339 & 1575 & 280 & 1.53 & 39 & 5 \\
\hline $12-071$ & 300 & 335 & 2.24 & 78.1 & 6 & $21-784$ & 955 & 162 & 1.08 & 29.8 & 3 \\
\hline $12-083$ & 280 & 387.5 & 1.20 & 52 & 6 & 21-923 & 1310 & 996 & 9.66 & 438.82 & 4 \\
\hline $12-085$ & 465 & 195.4 & 0.54 & 51.05 & $\infty$ & 21-924 & 1311 & 379.5 & 1.90 & 69.6 & 4 \\
\hline $13-038$ & 1800 & 248 & 0.62 & 40 & $\infty$ & $21-105$ & 1800 & 901 & 4.21 & 37.97 & 3 \\
\hline $47-015$ & 1040 & 3209 & 7.75 & 320 & 5 & $47-005$ & 1924 & 1910 & 1.13 & 72 & 6 \\
\hline $47-023$ & 1280 & 360 & 0.22 & 22 & $\infty$ & $47-007$ & 1814 & 587 & 4.36 & 34.2 & 4 \\
\hline $19-049$ & 2068 & 36 & 0.11 & 5.27 & 6 & 18-019 & 135 & 126 & 2.07 & 75 & 6 \\
\hline $19-053$ & 1440 & 1070.6 & 3.61 & 142.48 & 7 & $18-081$ & -20 & 780.5 & 21.33 & 415.1 & 11 \\
\hline $19-055$ & 1332 & 1638 & 2.60 & 56.6 & 3 & $18-083$ & -20 & 441.8 & 10.68 & 266 & 7 \\
\hline $19-063$ & 1420 & 98.1 & 0.78 & 116 & $\infty$ & $18-087$ & -22 & 355 & 5.57 & 125 & 7 \\
\hline $19-065$ & 780 & 7311 & 8.09 & 185.2 & 5 & $18-089$ & -23 & 206.2 & 6.89 & 167 & 8 \\
\hline $19-069$ & 705 & 11267 & 15.19 & 232 & 4 & 18-091 & -19 & 341.1 & 4.55 & 66 & 8 \\
\hline 19-097 & 1352 & 44 & 0.13 & 5.11 & 6 & 18-093 & -19 & 318.3 & 3.59 & 360 & $\infty$ \\
\hline 19-099 & 1459 & 40 & 0.08 & 2.11 & 5 & $18-095$ & -15 & 100.3 & 1.93 & 103 & $\infty$ \\
\hline $19-101$ & 1290 & 4003.7 & 6.28 & 351.4 & 5 & $21-145$ & 1780 & 615 & 3.37 & 99.5 & 6 \\
\hline 19-119 & 334 & 710 & 0.16 & 66.3 & $\infty$ & $21-163$ & 880 & 568 & 1.45 & 212 & $\infty$ \\
\hline $19-121$ & 820 & 156 & 0.16 & 18.6 & $\infty$ & $21-167$ & 1770 & 270 & 2.71 & 104.61 & 5 \\
\hline $19-148$ & 1800 & 8 & 0.07 & 0.949 & 4 & $21-171$ & 1520 & 773 & 7.70 & 125.37 & 5 \\
\hline 13-019 & 400 & 1256 & 6.26 & 108.91 & 8 & $21-177$ & 820 & 6700 & 41.86 & 923 & 5 \\
\hline $13-025$ & 270 & 2715 & 10.12 & 321 & 8 & 21-179 & 800 & 800 & 3.50 & 472.25 & $\infty$ \\
\hline $14-022$ & 570 & 524 & 2.31 & 46.7 & 4 & $21-181$ & 790 & 1108 & 1.44 & 142 & 6 \\
\hline $24-021$ & 1585 & 415 & 1.82 & 264 & $\infty$ & $21-183$ & 650 & 9140 & 48.33 & 945 & 6 \\
\hline $24-023$ & 1376 & 1410 & 3.75 & 665 & $\infty$ & $21-259$ & 2000 & 60.4 & 2.27 & 37.12 & 5 \\
\hline $24-027$ & 384 & 4300 & 10.13 & 671 & 6 & $21-261$ & 1490 & 1000 & 5.72 & 119 & 5 \\
\hline $24-065$ & 222 & 18525 & 19.64 & 1825.27 & 5 & $21-267$ & 1450 & 3400 & 15.85 & 445 & 5 \\
\hline 29-011 & 778 & 2420 & 2.57 & 1922 & $\infty$ & $21-279$ & 1450 & 2655 & 9.36 & 427.5 & 4 \\
\hline 44-015 & 651 & 3670 & 2.39 & 1019 & $\infty$ & $21-954$ & 1720 & 166 & 2.68 & 34.6 & 4 \\
\hline $52-005$ & 1158 & 1350 & 0.24 & 60 & $\infty$ & $21-966$ & 1420 & 234 & 1.35 & 78.16 & 5 \\
\hline $21-329$ & 1493 & 111 & 1.58 & 38.83 & 3 & 24-029 & 70 & 36500 & 38.94 & 2866 & 6 \\
\hline
\end{tabular}

climates (dry and wet regions, respectively) in Iran (see Figure 2). This condition occurs with a number of other couples of stations, such as 47-023 and 24021. In contrast, two other stations (i.e., 18-091 and 18-093) which are located near each other with similar climates, elevations, and areas have different correlation dimension values. Some important points can be concluded from these results. Firstly, Parameter $D_{2}$ can be used to identify inherent characteristics of the time series that are different due to the prevailing conditions in every catchment and can be used to determine the similarity or dissimilarity of the two considered stations. Secondly, the similarity in the location or climate of the stations does not result in similarities in their systems' behaviours.

As already mentioned, the correlation dimension values contain important information about the behaviours of the fundamental system dynamics that could be distinguished between catchment hydrological behaviours. Therefore, the correlation dimension values can be used as base criteria for the foundation of a catchment classification pattern. In this way, catchments are categorized into groups and subgroups using their similar hydrologic behaviours, which, in 


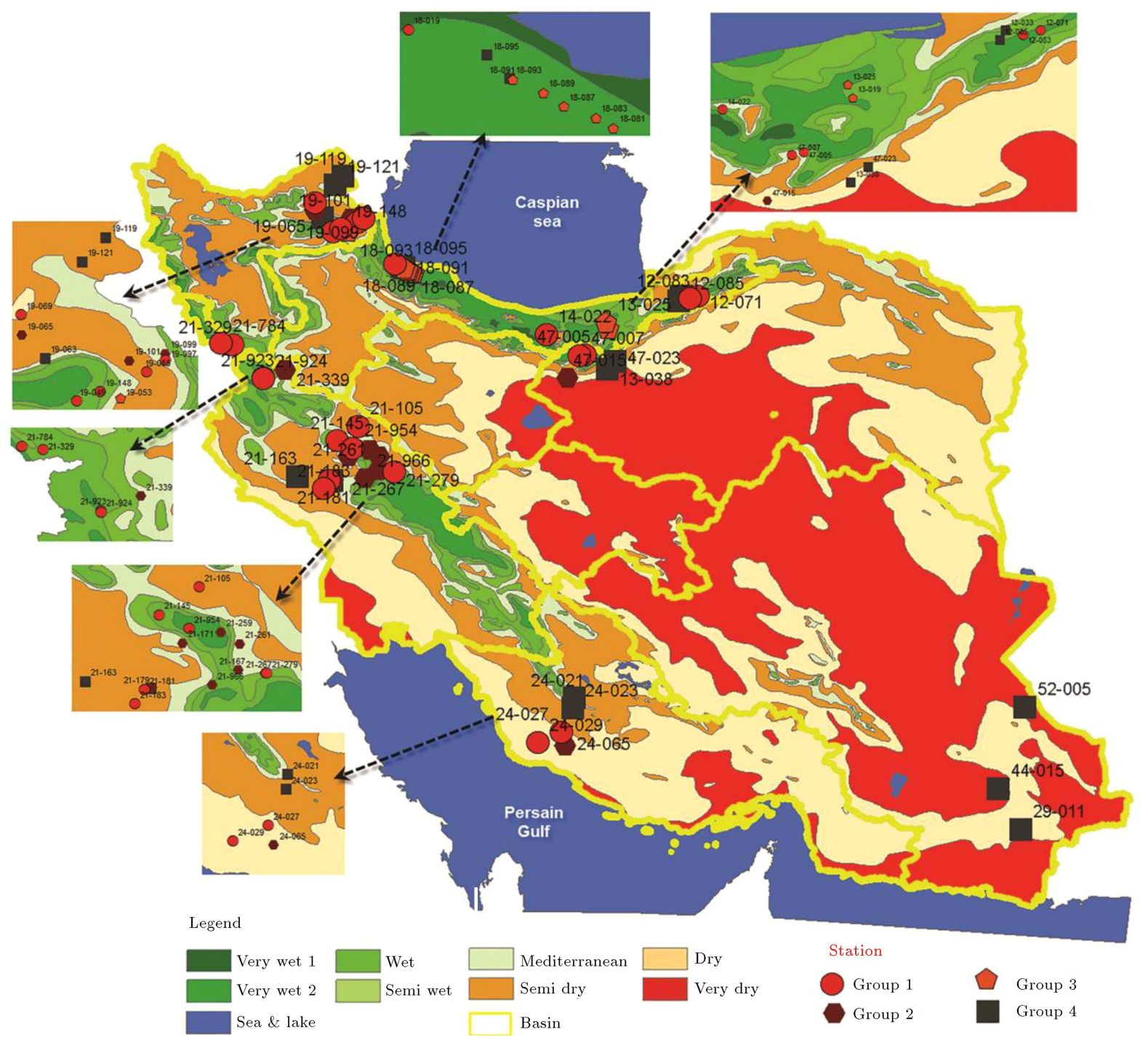

Figure 2. Scattering map of study stations and classification map.

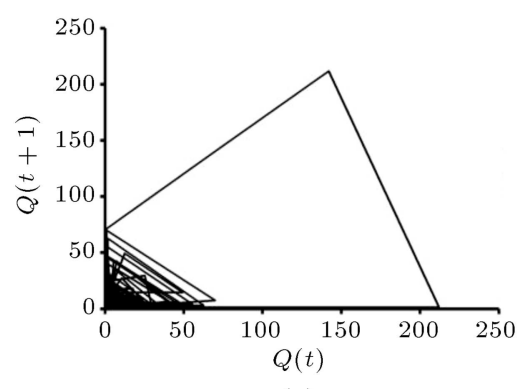

(a)

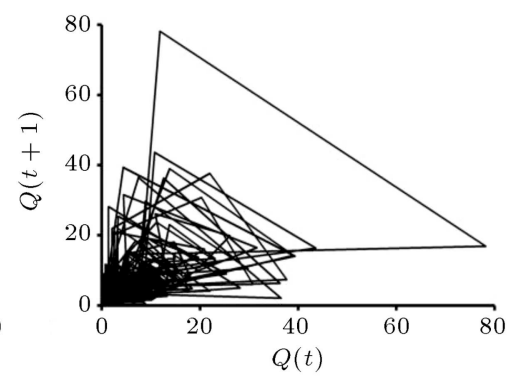

(b)

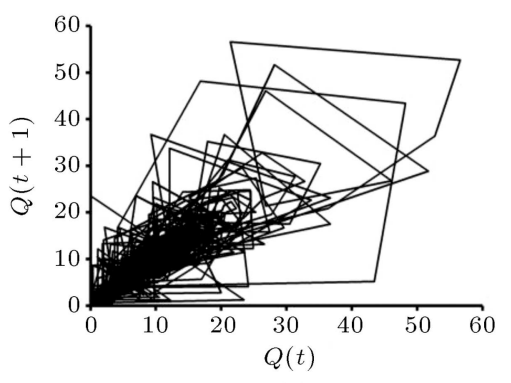

(c)

Figure 3. Phase space diagram for three selected stations: (a) 21-163, (b) 21-966, and (c) 19-055.

turn, are specified by correlation dimensions calculated for monthly outflow data.

Concerning the dimensionality of the studied stations in Table 1 , it can be seen that the catchments in the study area range widely from stochastic (undefined) to deterministic (low dimensions). Stations in the study area can be divided into four groups based on the correlation dimensions: low dimension $\left(D_{2}<=4\right)$ as Group 1, medium dimension $\left(D_{2}=5\right)$ as Group 2, high dimension $\left(D_{2}=>6\right)$ as Group 3, and unidentifiable as Group 4. The group's number and dimension value ranges are somewhat arbitrarily selected. However, the limit of the dimension values defined for each group is reasonable, because too many groups with very small 


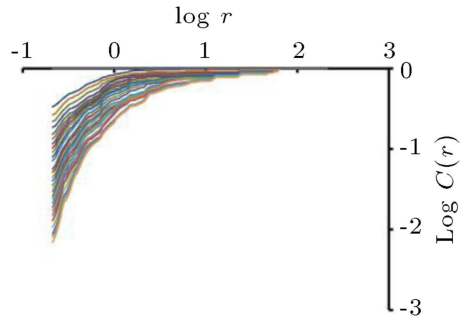

(a)

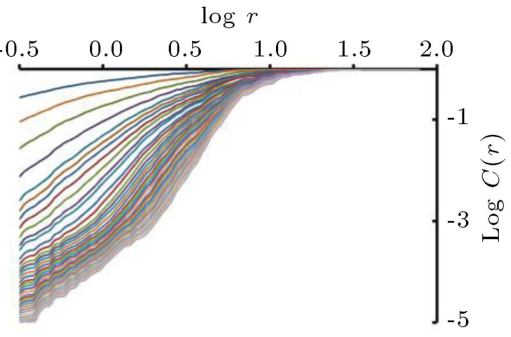

(b)

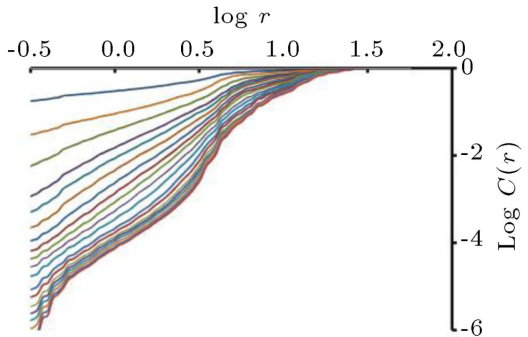

(c)

Figure 4. $\log C(r)$ versus $\log (r)$ plots for daily river flow data for three selected stations: (a) 21-163, (b) 21-966, and (c) 19-055.

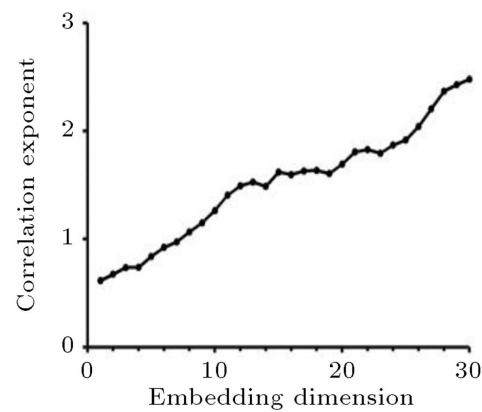

(a)

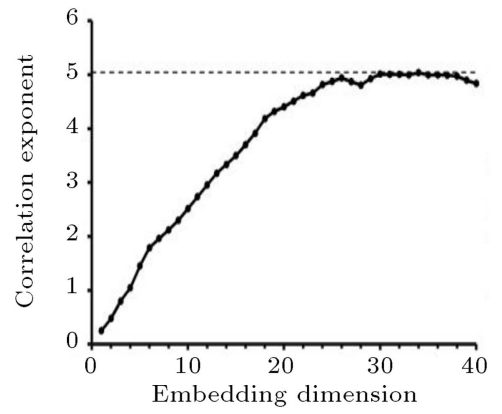

(b)

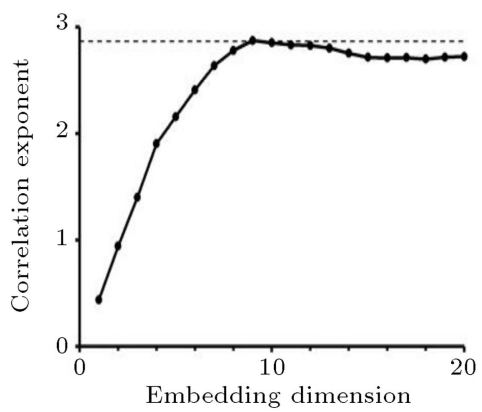

(c)

Figure 5. Relation between correlation dimension and embedding dimension for three selected stations: (a) 21-163, (b) 21-966, and (c) 19-055.

differences or only two groups presenting just high- and low-dimension values are not efficient for the purpose of classification.

Figure 2 presents the classification of stations in the study area into four mentioned groups, indicating the variability and patterns across the study area. The number of stations is not large enough, and more data are needed to analyse the regional results confidently; however, the stations are scattered all over the country with an element of homogeneity among them.

Based on Figure 2, a clear relationship between the correlation dimension and the spatial pattern cannot be established; however, a general view of the spatial pattern of groups and similarities is detectable. In southeast of Iran, the stations are classified as Group 4. The stations located in the south part with a semi-dry climate belonging to Group 4 decrease in group number (degree of complexity) as you move closer to the sea and dry climates. Moreover, none of the stations in the south part of Iran belongs to Group 1. Overall, the stations located in the west part of Iran mostly belong to Groups 1 and 2. Although all of the groups are observed in the northwest, most stations with wet or very wet climates in this region belong to Group 1. Most stations with wet (very wet, wet, and semi-wet) climates that are located in the north part of Iran are classified as Group 1.

Evident from an analysis in this study is that while the runoff dynamic of the south eastern stations exhibits very high complexity, more stations located in very wet climates (especially, in the north part of Iran) are less complex than the other stations. The tributaries of the south eastern rivers, unlike the north western rivers, are mostly seasonal. Thus, changes in discharges of the south eastern rivers are very considerable which lead to increasing their system's complexity. Furthermore, the climate changes resulting from the Oman sea currents affect the rivers flow significantly. In general, a decrease in rainfall (drought increasing) leads to an increase in the complexity value.

As mentioned, one of the advantages of this classification pattern is selecting the appropriate model before starting the modelling operation. In other words, by determining the classification group, the user can predict the appropriate model such as stochastic, neural network, etc. At this stage, it is required that the behaviours of different time series be compared with those of other groups; considering the model, researchers, in addition to presenting the accuracy criteria, should propose the classification criteria so that other researchers can specify which model is appropriate for which complexity level (or classification group). As an example, Tongal and Berndstoon [30] studied the impact of streamflow complexity on the forecasting result of three types of models (chaos, stochastics, and black-box). They showed that determining the degree of complexity could help with the pre-determination of model to be applied. Therefore, the proposed classification framework can be a proper candidate for selecting the type and quantity of the 


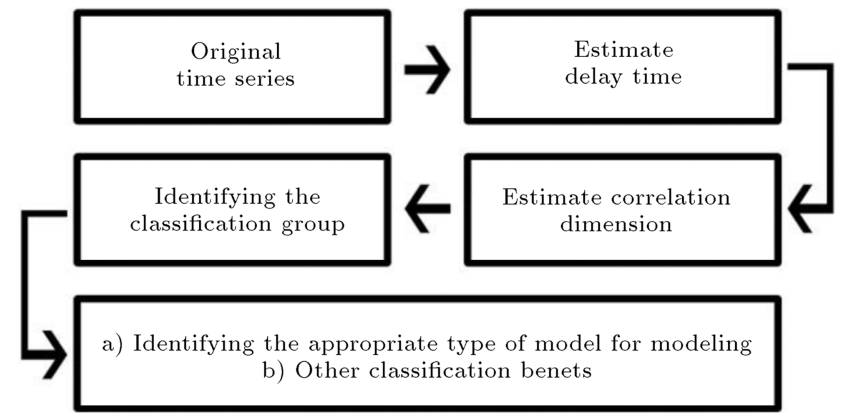

Figure 6. Schematic representation of the proposed classification pattern.

model. While more than six effective variables $\left(D_{2} \geq\right.$ 6) may result in high complexity requiring the use of more complex models, four or fewer variables $\left(D_{2} \leq 4\right)$ lead to lower complexity, which can be analysed by simpler models. As an example, the obtained results in this research suggest that the stochastic models could be a first-order selection to model the south eastern stations in Iran.

Figure 6 shows how to use the classification pattern. Accordingly, $D_{2}$ is calculated after calculating the delay time; based on its value, the time series in classification is specified. By specifying times series, this classification can be used in cases such as selecting the appropriate model for the time series modelling (and other classification benefits).

\section{Conclusion}

An important hydrological challenge is to establish a catchment classification framework which serves as a basis for many purposes in hydrology (e.g., for generalization and developing models). In this regard, finding a sensitive and interpretable criterion is required that can be used for distinguishing various catchments properly. In this study, the capability of the chaos theory using the correlation dimension value $\left(D_{2}\right)$ is tested for this purpose.

The results of this study showed that the application of the correlation dimension is suitable for assessing the differences in catchment behaviours (runoffs) resulting from various climatic and hydrological conditions. Based on the discussed results, this criterion can be used as an index or a measure to obtain a catchment classification framework. In the present study, the result of the dimensional analysis is used to categorize Iran's catchments into four groups based on the degree of time series complexity: low dimensional (low complexity), medium dimensional (medium complexity), high dimensional (high complexity), and unidentifiable (very high complexity or stochastic).

The spatial pattern classification of Iran's catchments indicates that the catchments with different climate characteristics, which are located at a far distance from each other, might yield similar responses along with the same degree of complexity. Furthermore, spatial plot results showed some degree of homogeneity and connectivity between catchments.

The presented classification method in this study can be used by engineers and researchers to study hydrological modelling methods more effectively through matching the degrees of complexity between models and catchment responses (streamflow time series).

In future studies, finding a new approach that can suggest definitive guidelines to specify the boundaries of groups and sub-groups in a classification framework is necessary. In addition, the efficiency of classification in an existing model could be studied. The time scale plays one of the most important roles in dimensionality. Thus, every time scale could be studied to identify the group and sub-group boundaries. The ability of these criteria must be tested with different climate and catchment properties in other countries. For future study, it is suggested that the results of this study be compared and validated with those of another classification method. Applying the method under climate change conditions and multivariate chaotic method in order to optimize the obtained results using data of precipitation, runoff, sediment, etc. can be another issue. We emphasize that the finding of "similarity measures" or an agreed-upon "classification framework" should come first and develop more.

\section{References}

1. McDonnell, J.J. and Woods, R.A. "On the need for catchment classification", Journal of Hydrology, 299, pp. 2-3 (2004).

2. Gani, A., Siddiqa, A., Shamshirband, S., and Hanum, F. "A survey on indexing techniques for big data: taxonomy and performance evaluation", Knowledge and Information Systems, 46, pp. 241-284 (2016).

3. Grigg, D.B. "The logic of regional systems", Annals of the Association of American Geographers, 55, pp. 465-491 (1965).

4. Wagener, T., Sivapalan, M., Troch, P.A. and Woods, R.A. "Catchment classification and hydrologic similarity", Geography Compass, 1(4), pp. 901-931 (2007).

5. Olden, J.D., Kennard, M.J., and Pusey, B.J. "A framework for hydrologic classification with a review of methodologies and applications in ecohydrology", Ecohydrology, 5, pp. 503-518 (2011).

6. Sivakumar, B. and Singh, V.P. "Hydrologic system complexity and nonlinear dynamic concepts for a catchment classification framework", Hydrology and Earth System Sciences, 16, pp. 4119-4131 (2012).

7. Zhang, Y., Xia, J., Bunn, S.E., Arthington, A.H., Mackay, S., and Kennard, M. "Classification of flow regimes for environmental flow assessment in regulated 
rivers: the Huai River Basin, China", River Research and Applications, 28, pp. 989-1005 (2011).

8. Bejarano, M.D., Marchamalo, M., De Jalón, D.G., and De Tánago, M.G. "Flow regime patterns and their controlling factors in the Ebro basin (Spain) ", Journal of Hydrology, 385, pp. 323-335 (2010).

9. Monk, W.A., Wood, P.J., Hannah, D.M., Wilson, D.A., Extence, C.A., and Chadd, R.P. "Flow variability and macro invertebrate community response within riverine systems", River Research and Applications, 22, pp. 595-615 (2006).

10. Rao, A.R. and Srinivas, V.V. "Regionalization of watersheds by fuzzy cluster analysis", Journal of Hydrology, 318(1), pp. 57-79 (2006).

11. Zoppou, C., Nielsen, O.M., and Zhang, L. "Regionalization of daily streamflow in Australia using wavelets and $\mathrm{K}$ means analysis, CMA Research Report MRR02-003", Australian National University, Canberra. Available at: http://wwwmaths. anu.edu.au/research.reports/mrr/02/003/ (2002).

12. Krasovskaia, I. "Entropy-based grouping of river flow regimes", Journal of Hydrology, 202, pp. 173-191 (1998).

13. Sen, A.K. "Complexity analysis of river flow time series", Stochastic Environmental Research and Risk Assessment, 23, pp. 361-366 (2009).

14. Chou, C. "Complexity analysis of rainfall and runoff time series based on sample entropy in different temporal scales", Stochastic Environmental Research and Risk Assessment, 28, pp. 1401-1408 (2014).

15. Sivakumar, B. "Dominant processes concept in hydrology: moving forward", Hydrological Processes, 18, pp. 2349-2353 (2004).

16. Sivakumar, B., Jayawardena, A.W., and Li, W.K. "Hydrologic complexity and classification: a simple data reconstruction approach", Hydrological Processes, 21, pp. 2713-2728 (2007).

17. Sivakumar, B., Woldemeskel, F.M., and Puente, C.E. "Nonlinear analysis of rainfall variability in Australia", Stochastic Environmental Research and Risk Assessment, 28, pp. 17-27 (2014).

18. Vignesh, R., Jothiprakash, V. and Sivakumar, B. "Streamflow variability and classification using false nearest neighbour method", Journal of Hydrology, 531, pp. 706-715 (2015).

19. Hrachowitz, M., Savenije, H.H.G., Blöschl, G., McDonnell, J.J., Sivapalan, M., Pomeroy, J.W., Arheimer, B., Blume, T., Clark, M.P., Ehret, U., Fenicia, F., Freer, J.E., Gelfan, A., Gupta, H.V., Hughes, D.A., Hut, R.W., Montanari, A., Pande, S., Tetzlaff, D., Troch, P.A., Uhlenbrook, S., Wagener, T., Winsemius, H.C., Woods, R.A., Zehe, E., and Cudennec, C. "A decade of predictions in ungaged basins (PUB) - a review", Hydrological Science Journal, 58, pp. 1198-1255 (2013).
20. Sivakumar, B., Singh, V.P., Berndtsson, R., and Khan, S.K. "Catchment classification framework in hydrology: Challenges and directions", Journal of Hydrologic Engineering, 20(1), 4014002-4014002 (2015).

21. Liu, Q., Islam, S., Rodriguez-Iturbe, I., and Le, Y. "Phase-space analysis of daily streamflow: characterisation and prediction", Advances in Water Resources, 21, pp. 463-475 (1998).

22. Fan, Q., Wang, Y., and Zhu, Li. "Complexity analysis of spatial-temporal precipitation system by PCA and SDLE", Applied Mathematical Modelling, 37, pp. 40594066 (2013).

23. Takens, F. "Detecting strange attractors in turbulence", Lecture Notes in Mathematics, 898, pp. 366381 (1981).

24. Grassberger, P., and Procaccia, I. "Measuring the strangeness of strange attractors", Physica D: Nonlinear Phenomena, 9(2-1), pp. 189-208 (1983).

25. Ghorbani, M.A., Kisi, O., and Aalinezhad M. "A probe into the chaotic nature of daily streamflow time series by correlation dimension and largest lyapunov methods", Applied Mathematical Modelling, 34, pp. 4050-4057 (2010).

26. Schertzer, D., Tchiguirinskaia, I., Lovejoy, S., Hubert, P., and Bend-joudi, H. "Which chaos in the rainfallrunoff process? A discussion on "Evidence of chaos in the rainfall-runoff process by Sivakumar et al.", Hydrological Sciences Journal, 47, pp. 139-147 (2002).

27. Sivakumar, B. "Correlation dimension estimation of hydrologic series and data size requirement: myth and reality", Hydrological Sciences Journal, 50, pp. 591604 (2005).

28. Sivakumar, B., Berndtsson, R., Olsson, J., and Jinno, K. "Reply to "Which chaos in the rainfall-runoff process?" by Schertzer et al.", Hydrological Sciences Journal, 47, pp. 149-158 (2002).

29. Sivakumar, B., Persson, M., Berndtsson, R., and Uvo, C.B. "Is correlation dimension a reliable indicator of low-dimensional chaos in short hydrological time series?", Water Resources Research, 38(2), pp. 1-8 (2002).

30. Tongal, H. and Berndtsson, R. "Impact of complexity on daily and multi-step forecasting of streamflow with chaotic, stochastic, and black-box models", Stochastic Environmental Research and Risk Assessment, 31(3), pp. 661-682 (2017).

\section{Biographies}

Hadi Delafrouz is a PhD candidate at Iran University of Science and Technology, School of Civil Engineering. He obtained his BS degree from Shahrood University in 2000 and MS degree from Tabriz University in 2007. His research area lies in hydrological, hydraulic modelling and catchment management. At present, he is actively involved in applying complexity concept 
in catchment classification. He has published 14 national and international journals, and conference proceedings.

Abbas Ghaheri is a Professor at Iran University of Science and Technology, School of Civil Engineering, where he has earned academic experience from 1976 until now. He obtained his BS degree from Tehran University, Iran, and MS and PhD degrees from Colorado State University, USA. His research interests include design of run off collection network, river mechanics and flood plain analysis and management, water resources development, planning, and management, ground water analysis, and planning for ground water supply. The results of his research have been presented in over 80 publications in international journals, book chapters, conference proceedings, and research reports. He has also advised and supervised numerous projects.

Mohammad Ali Ghorbani is an Associate Professor of Hydrology at the University of Tabriz, where he firstly joined in 2006. His main research focuses on hydrology, water resources and artificial intelligence, etc. He has more than 10 years of research experience and has guided PhDs and MScs in the field of Hydrology. He received his BSc in Irrigation from University of Tabriz in 1997, and MSc in Hydraulic Structures from University of Tehran in 2000. He received his $\mathrm{PhD}$ in Hydrology from University of Tehran in 2006. He has published 54 international and 60 national peerreviewed papers, one chapter in an international book, and two books in Persian. 